\title{
An Analysis of "How Much Is an NBA Ticket Worth in China Now" from the Perspective of Multi-Modal Intertextuality
}

\author{
Yang Yang \\ School of Foreign Languages, East China University of Science and Technology, Shanghai, China \\ Email: 1281742379@qq.com
}

How to cite this paper: Yang, Y. (2021) An Analysis of "How Much Is an NBA Ticket Worth in China Now" from the Perspective of Multi-Modal Intertextuality. Open Access Library Journal, 8: e6896. https://doi.org/10.4236/oalib.1106896

Received: October 13, 2020

Accepted: March 22, 2021

Published: March 25, 2021

Copyright $\odot 2021$ by author(s) and Open Access Library Inc.

This work is licensed under the Creative Commons Attribution International License (CC BY 4.0).

http://creativecommons.org/licenses/by/4.0/

\section{(c) (i) Open Access}

\begin{abstract}
This paper analyzes the multi-modal text "How Much is An NBA Ticket Worth in China Now" from the perspective of intertextuality. Based on the theories of intertextuality and cultural intertextuality, four story lines in multi-modal texts are extracted. The story lines clearly show that Daryl Morey's comments about Hong Kong have disappointed Chinese fans and, in turn, caused the NBA to lose a lot of the Chinese market. After exploring these details, this paper abstracts the root causes of contradictions in the multi-modal text from the perspectives of Chinese national feelings, the realistic function of sports diplomacy and the difference view of freedom of speech. Finally, based on these findings, a questionnaire has been designed and SPSS was used to verify the reliability. After collecting data, we verified the realistic reasons behind the storyline, in order to make suggestions for Sino-American cultural exchanges.
\end{abstract}

\section{Subject Areas}

Culture, Linguistics

\section{Keywords}

Multi-Modal Text, Intertextuality, Speech Freedom, Patriotism

\section{1. 引言}

新媒体的发展促使多模态文本层出不穷。分析多模态文本，除了分析语 言文字本身之外，还注重研读图像、声音、颜色等多种传递意义的手段和符 号资源。在对多种模态进行分析时，观众对其他文本的印象也时常被唤起， 文本与文本间发生互动, 产生互文性。将互文性运用到多模态文本分析不仅 
有利于作者本身意义的传达, 也在一定程度上加深了观众的情感认知。本文 将以北京周报的双语视频 How Much is An NBA Ticket Worth in China Now? ${ }^{1}$ 为语料, 研究多模态文本中互文性对故事线构建的作用, 客观地还原事件真 相及意识形态的差异。

\section{2. 文献综述}

多模态话语就其性质而言是人类感知通道在交际过程中综合使用的结果 (朱永生 2007) [1]。20 世纪 90 年代, Kress \& van Leeuwen [2]首先提出 “多模 态话语” 这一概念, 语言学界兴起了对多模态的研究。在系统功能语法下, 二人又第一次系统的提出了视觉语法概念。以语言元功能的概念意义, 人际 意义, 语篇意义为基础, 确立了 “再现意义” “互动意义” 和 “构图意义” 的视觉图像分析理论(许晓翠 2019) [3]。从图像的各个方面来研究多模态活 动, 从而对图像进行比较全面系统的分析。李战子(2003) [4]对二人的理论进 行解读后, 我国也如火如茶的开展了多模态话语分析。多模态话语的最合适 的理论模式是系统功能语言学理论。基于此, 张德禄(2015) [5]建构了由四个 层面组成的多模态话语分析综合框架, 即利用媒体层面实现媒体形式(表达层 面)、在形式层面由探讨构成意义层面的各种模态(内容层面); 文化层面制约 语境层面, 语境推进内容层面的形成, 故而, 内容层面是文化层面的体现。

互文性是法国符号学家克里斯特娃(1982) [6]提出的。即 “任何作品的文 本都是像许多行文的镶嵌品那样构成的, 任何文本都是其他文本的吸收和转 化。它们相互参照, 彼此牵连, 形成一个潜力无限、开放的动态网络。” 邵 长超(2015: 125) [7]认为: “互文性是一个指涉体系, 各种关系的实现是通过符 号互动完成的。研究步骤可从词、词组、分句、句群到文本、话语。”互文 这一概念应用极为广泛, 与广义概念上的互文不同的是, 热奈特(1977) [8]在 互文性研究方面确立了文本类属, 基于文本间的联系和转化, 构成了更详细 具体的互文性研究框架。通过梳理互文性基本理论, 文章发现互文性不仅可 以表现两个或两个以上文本间发生的互文关系; 其作为一种文本理论，也可 用于语篇分析。

多模态与互文为何可以结合? 张春燕 (2015) [9]认为语境(即层面)理论为 多模态话语分析与互文性结合分析提供理论基础。侯建波(2016) [10]认为索绪 尔关于符号的能指和所指理论是多模态语篇的基础。所指对应现实世界的事 物和过程; 能指对应符号系统,包括声音、文字、图像等符号系统。所以多模 态框架和社会符号学为二者结合提供可能。由于多模态话语分析现在研究内 容主要是静态文本为主, 动态多模态话语为辅, 再加之传统的互文性分析多 聚焦于单一模态即文字文本间的互文, 而少有人探讨动态多模态话语间的互 文现象。本文研究的是四分钟的动态文本, 分析形式层面中主要的图觉和语 言。在研读了多篇文献后笔者发现一般的多模态互文局限于同一文本的不同 模态, 所以本文将试着从外互文性(即不同文本间的关系)、文化互文的角度, 解读图像之间的联系, 抽取动态文本中的故事线, 进而发现推动故事线发展 
的缘由。

\section{3. 多模态互文分析}

以反修例为借口, 2019 年 6 月香港出现暴力事件并不断持续, 在网络上, 不少青年网友自发传播爱国爱港声音。“饭圈女孩” 集体 “守护全世界最好 的阿中(中国)” 呼吁内地和香港青年团结一致。在此背景下 10 月 5 日，休斯 顿火箭队总经理达雷尔 - 莫雷在推特上发表了一条支持香港示威者的口号。

How Much is An NBA Ticket Worth in China Now? 是北京周报于 10 月 14 日 对此事件做出的官方评述。除了图 1 背景画面之外, 四分钟内还主要出现了 不同画面, 分别与其他文本, 视频构成互文。基于多模态文本内容的相关性, 我们将其解读为如下四条故事线, 其中故事线一侧重考量与外部文本互文, 促进了主文本信息的传递; 故事线二考察主文本不同模态间互动的同时，考 量一部分由外互文引起的冲突效果。故事线三, 故事线四侧重图像这一模态 达到的文化互文效果。

\section{1. 故事线一：NBA 相关言论引起粉丝不满}

根据张德禄对多模态话语形式及关系角度划分，这些图文都属于互补关 系中的强化, 即图觉对字幕或者主持人解读音频的一种强化。鉴于第一条故 事线的侧重点, 我们将字幕放在图像旁阐释。

图 1 是事件起因也是视频创作缘由。将莫雷支持香港暴乱的原文本置于 其中, 成为主视频的一个互文本, 其聚焦于推特的本身: 一张写着 “fight for freedom stand with Hong Kong” 的配图。支持自由无可厚非，但这句话是香 港极端暴力分子的口号。香港问题涉及中国领土及主权，香港极端分子做出 一系列目无法纪的残暴行为，他们践踏法治，威胁社会秩序，危害香港人民 的根本利益，公然挑战一国两制的底线。不同于和平示威活动，暴力分子大 肆破坏基础设施, 一言不合就威胁他人生命, 这更接近于恐怖行为, 是人性 的泯灭。他们是为自由而战吗? 不, 他们打着自由的幌子, 在外界势力的干

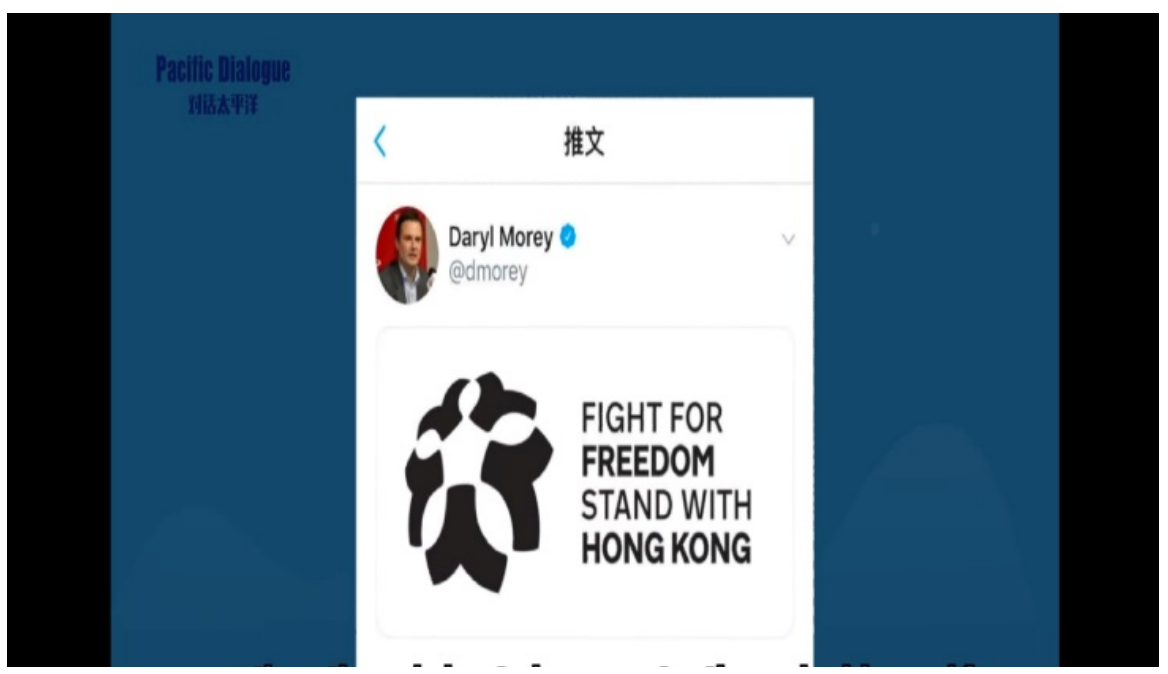

图 1. 莫雷发了一条推特, 支持香港暴行。 
涉下, 损害中国神圣的主权。莫雷作为 NBA 休斯敦火箭队的总经理, 影响力 巨大，他的言论于中国粉丝看来绝对超出了个人立场，故而他公然表态支持 乱港行径, 在中国引起一片哗然。与莫雷推特互文不仅唤起了观众对该事件 的记忆，更暗示了莫雷作为公众人物由于言论不当造成了不小的影响。

图 2-4 是粉丝的一系列反应。与图 2 产生互文的是其他视频，由一位知 名自媒体制作者在微博、bilibili 等社交软件上发布。视频名为我撕掉了价值 16,000 的球票中, 创作者解释了这样做的原因: 看 NBA 球赛是球迷梦㝝以求 的事情, 为此以 16,000 的高价实现梦想是值得的, 但是莫雷及 NBA 的相关 言论对创作者的三观产生了强大的冲击, 加之 NBA 不了解实际讯息而发声让 粉丝寒心，高价球票对于他而言现在的 “价值” 为负。创作者认为如何让负 价值为正呢? 那就是赋予它言语的权利, 让它承载中国粉丝的情感，即：体 育无国界但球迷有国籍，我们球迷首先是个中国人。该视频一发布，在 bilibili 上获得百万余投币，播放量在该平台为日排行榜最高第一名。在微博上播放 量一周内也达 655 万。与该视频在内容上外互文使粉丝行为更具有说服力,

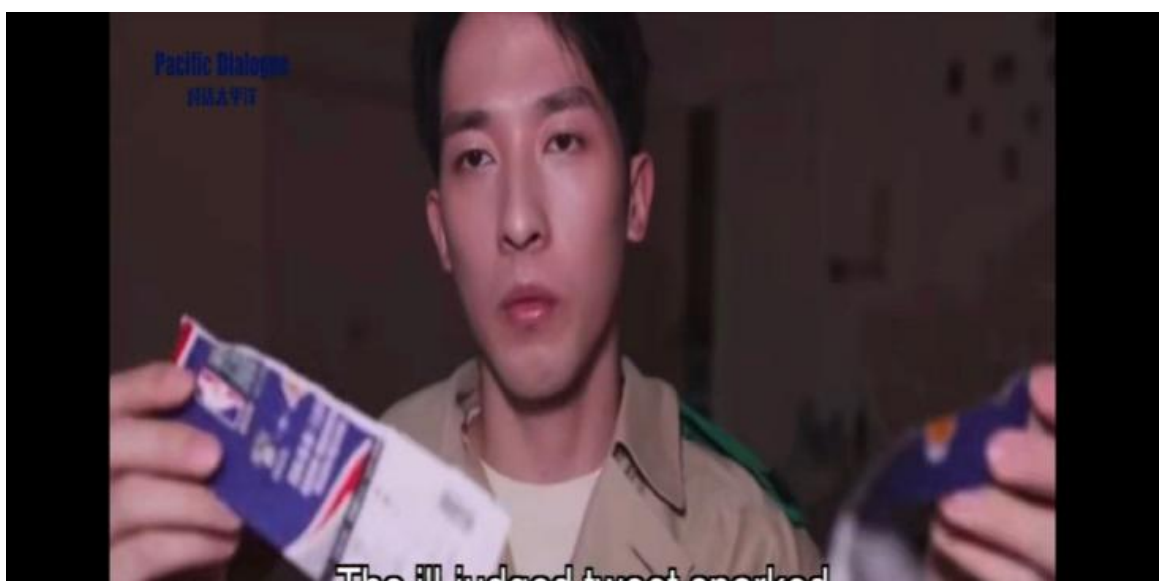

图 2. 这一有失偏颇的言论(即莫雷推特内容)立刻引发了网友的不满。(图为网友不满 的一个反应)

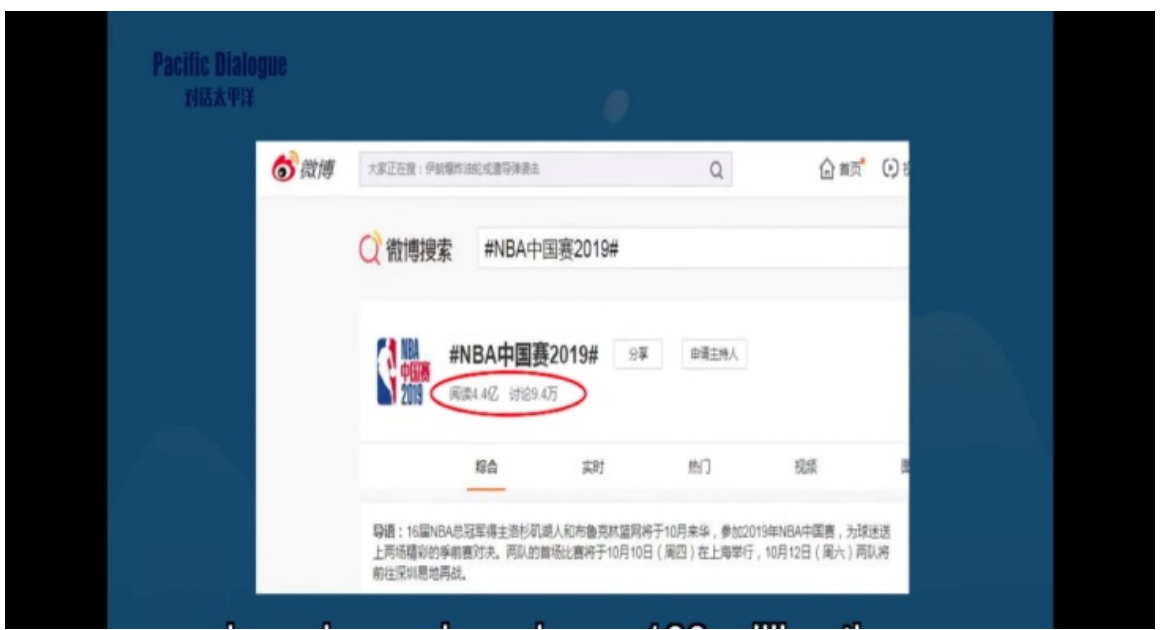

图 3. 在中国版 “推特” 微博平台上, 和 NBA 有关的话题汶览量几乎都在 1 亿以上。 大批网友留言表达他们的担忧和气愤。 


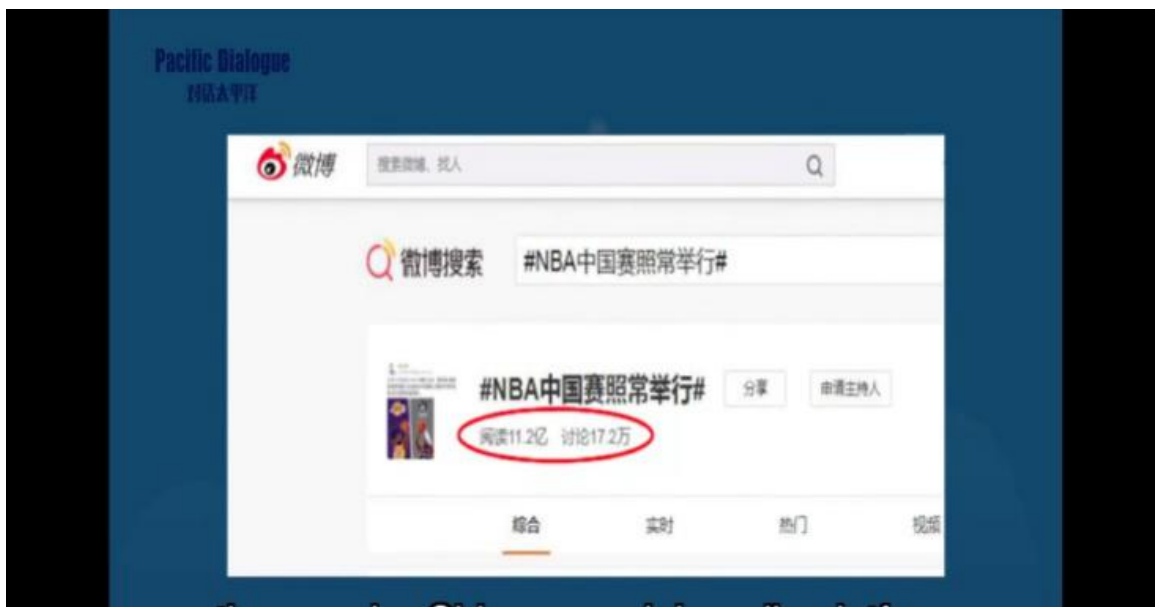

图 4. 在中国版 “推特” 微博平台上, 和 NBA 有关的话题汶览量几乎都在 1 亿以上。 大批网友留言表达他们的担忧和气愤。

形象也更为生动。视频内容与主文本的标题也产生了文本内的互动, 回答了 标题 NBA 球票到底价值几何。图 4、图 5 是该事件一出就产生的微博热议话 题。回顾背景莫雷言论发生在 2019NBA 中国赛几天之前, 时值中国 70 年国 庆阅兵典礼。高涨的爱国情绪和个人兴趣产生冲突时，人们毫无疑问需要支 持前者。图 4、图 5 的互文作用在于阐释了在事件背景下, 莫雷不负责任的 言论产生的直接效应，点明了主文本的谈论主题。

\section{2. 故事线二: 粉丝为何不满}

\subsection{1. 主文本内部阐释原因}

这条故事线的构造主要通过语言模态, 图像起了强化关系中的扩充作用。 对应功能语法中的概念功能, 图像表达相应的再现作用, 表征图像中事件的 概念关系，共同达到阐释作用。

同一模态之间构成链条产生互文，传递表层原因。图 5 与出现的 NBA 官 方声明图片互文, 构成 NBA 对莫雷事件的处理链条。此时文字解说构成的链 条是, NBA 总裁肖华认为 NBA 追求的是美国价值, 拒绝道歉。此时图像再 现意义与文本产生互动, NBA 不为对中国领土误读而道歉，作为中国人的球 迷该做何反应呢? 图 6 及接下来的一处文字互文却与之前的表面原因形成冲 突性互文, 第一处冲突在于 NBA 坚守美国价值, 却享有中国粉丝带来的市场 优势，获得实际的价值，而在利益价值面前，却不愿费心去了解中国顾客的 底线、尊重这片市场的主权，这种冲突是表层原因背后的故事。第二处冲突 体现在主持人对肖华言论的引用 “the power of sports can make a difference”。 肖华认为体育力量允许 NBA 对中国主权有不同解释, 但是主持人认为 NBA 作为强大的体育品牌, 它让中国粉丝失望的力量无比强大。这两处冲突性互 文将表层原因揭露开来, 不曾道歉是表象, 但真正不满的原因是 NBA 对体育 精神的论辩, 对粉丝敏感情绪的无视和㝇渎。

\subsection{2. 外部互文本阐释}

在解释原因时与外部文本互动, 更加客观和有说服力。与图 7 产生外互 


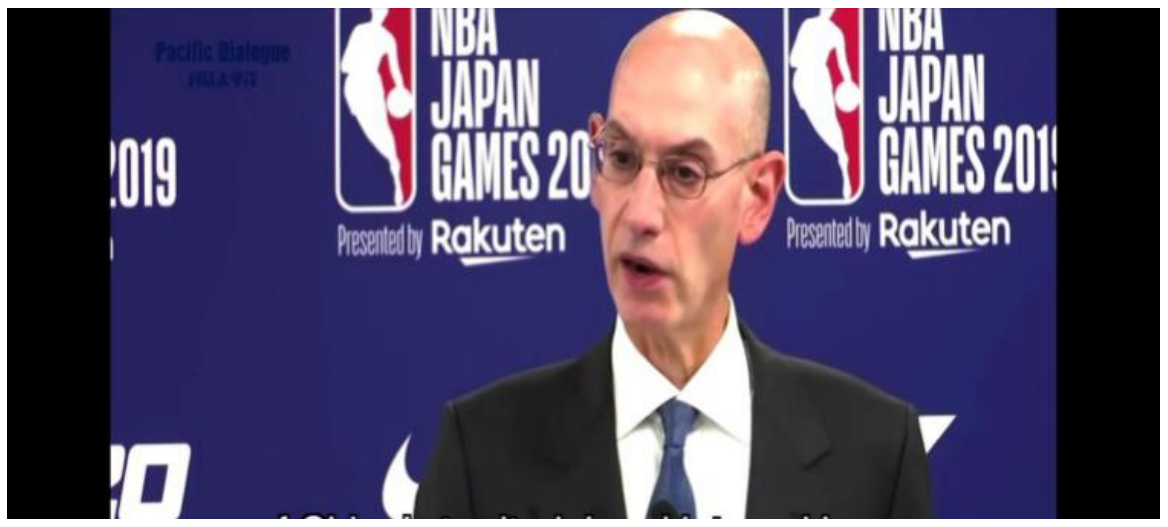

图 5. NBA 总裁就此事件发表声明却未道歉。

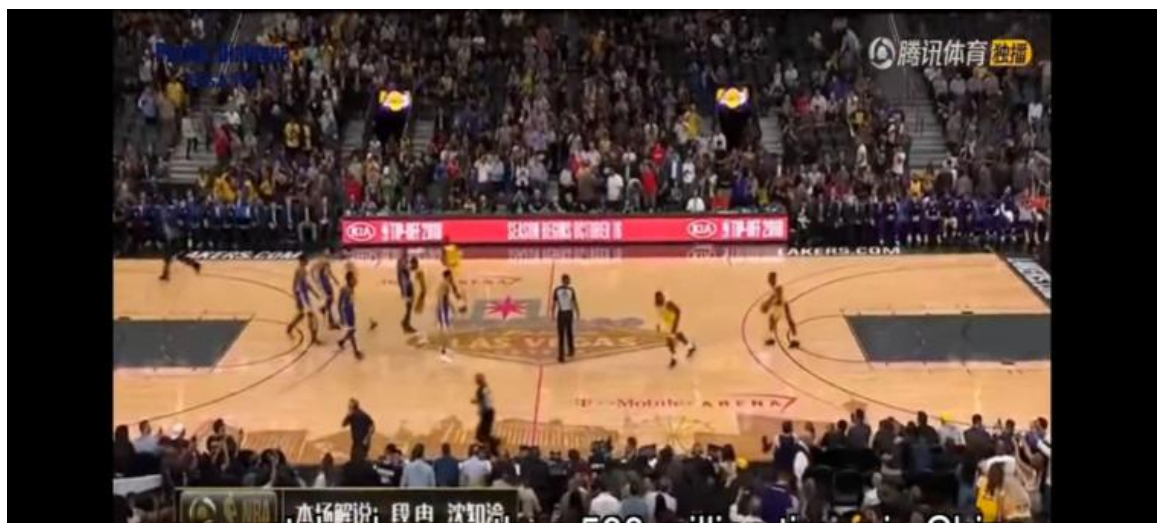

图 6. 2018 赛季观看及转播观看量达 5 亿多次。

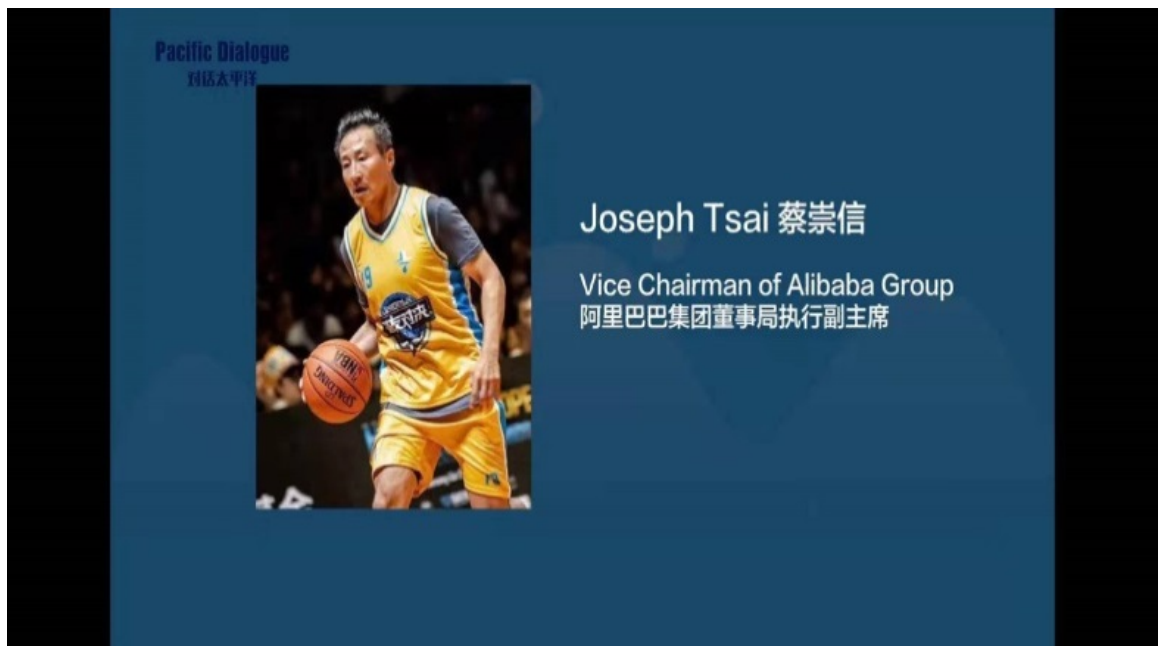

图 7. 华裔发声。

文的是蔡崇信对所有 NBA 球迷的公开信。蔡崇信, 一支 NBA 球队的老板, 深知中国文化的华裔坚定地站出来表达观点, 他解答了中国粉丝为何愤怒: 一、政治色彩伤害球迷情感。二、支持中国领土上发生的分离主义是中国人 的雷区。三、历史经历促使中国人在涉及领土意识时紧密的团结在一起。图 8 与 NBA 前老板蒂尔曼新书形成冲突性互文, 书名译为三思而后行, 但分开 


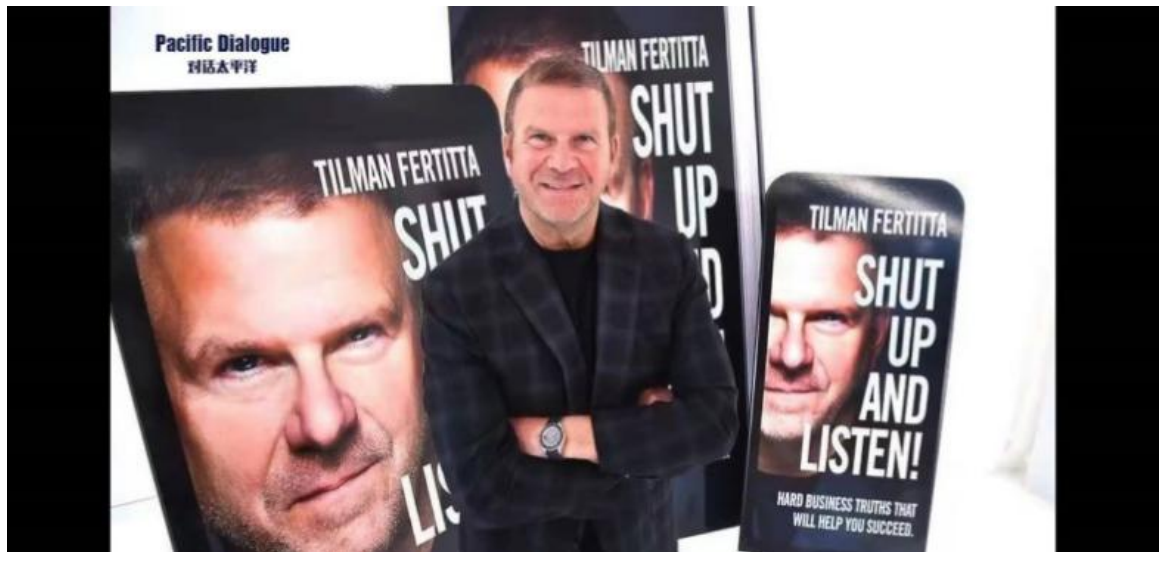

图 8. 休斯顿火箭队前老板的新书。

来译是, “闭嘴” 和 “听”, 这种巧妙地拆词让主文本生动的表达了观点: “中国并没有要求任何人闭嘴, 但也希望你们可以用心聆听”。值得一提的是 图 8 在构图上采取的是从中心到边缘的放置, 在互动意义上蒂尔曼直视镜头, 与观众形成接触, 这是 Kress \& van Leeuwen (2006) [11]定义的 “索取行为”。 当图像表示 “索取行为” 时, 图像中的表征参与者直视观看者, 表征对象与观 看者接触, 希望观看者做出某种行为或者与观看者建立某种联系。那在蒂尔曼 的直视下, 视频发布者希望观看者做出何种行为呢? 书名给予回答。这又在 某种程度上解析出来中国粉丝愤怒的原因, NBA 没有基本尊重且冷酷无情。

\section{3. 故事线三：西方媒体解读事件冲突}

如上, 三张图(图 9-11)的解说文本都是: 与中国相关的话题出现时, 一 些西方媒体总是用固定的模式来解读, 不是侵犯人权就是言论自由。《纽约 时报》(The New York Times)长期以来拥有良好的公信力和权威性, 在全世界 影响力巨大, 代表着美国高级报纸、严肃型刊物。在它中规中矩的报道中客 观理性的阐述了肖华的解读, 保护每个美国人的言论自由。该互文本的作用 在于对解说文本的证实。《卫报》(The Guardian)是英国全国性的综合内容报 纸，代表着独立性新闻，一般题目高度融合作者观点：外互文的图用疑问句 加强语气, 质疑了 NBA 声明: 分歧如何只归因于言论自由呢? 卫报认为是个 悖论。每日先驱报是英国自从上世纪 80 年代以来发行量最大的日报, 侧重于 社会类报道, 一贯吸人眼球。图 11 作用与图 9 一致。

所以何为美国人的人权和言论自由呢? 谈及人权, 多篇文章在分析案例 后得出, 受意识形态、政治霸权倾向等影响, 人权在美国具有强大的双重标 准, 尤其在涉及文化传统与社会制度差异上, 他们以高标准衡量别国, 以低 标准要求自己。那何为言论自由? 言论自由是指公民有权通过各种语言形式, 针对政治和社会中的各种问题表达其思想和见解的自由。关于言论自由这一 互文现象, 首先从主文本的文字模态看, 言论自由需要知识背景, 否则只能 为噪音。这表明莫雷可以自由发言, 但是不等于是非不分。基于黑白颠倒的 言论自由毫无意义。其次从互文本 CGTV 看, 美国的双重标准再次作崇。斯 特林是原 NBA 快船球队老板, 却因 2014 年公开发表种族歧视言论, 本人被 

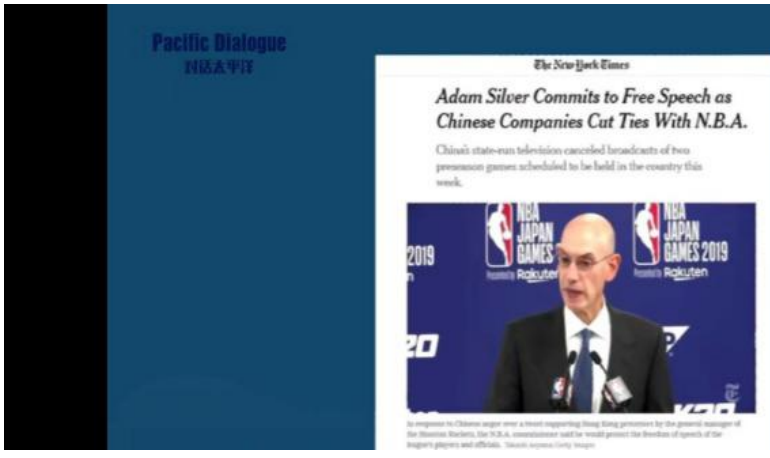

图 9. 纽约时报。

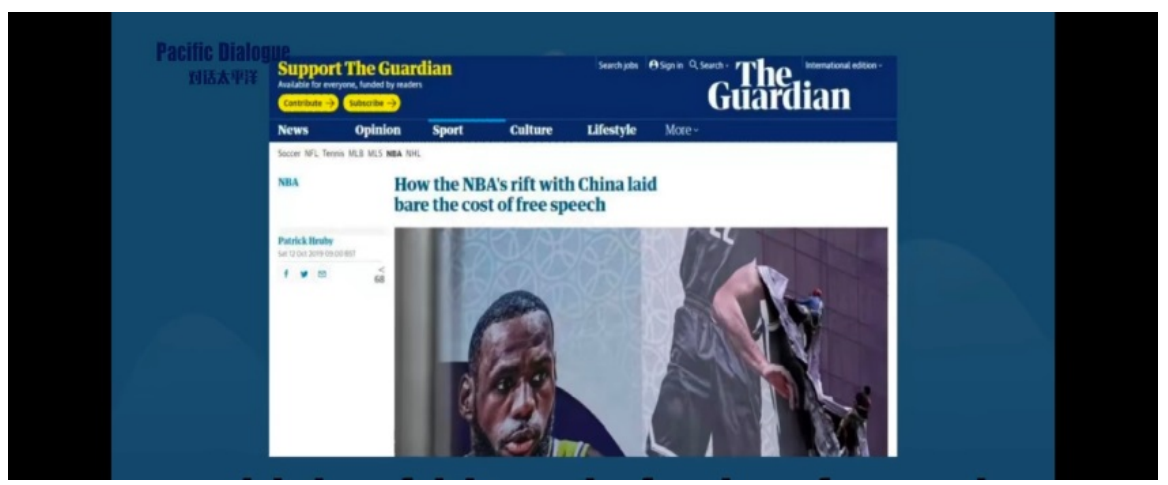

图 10. 英国卫报。

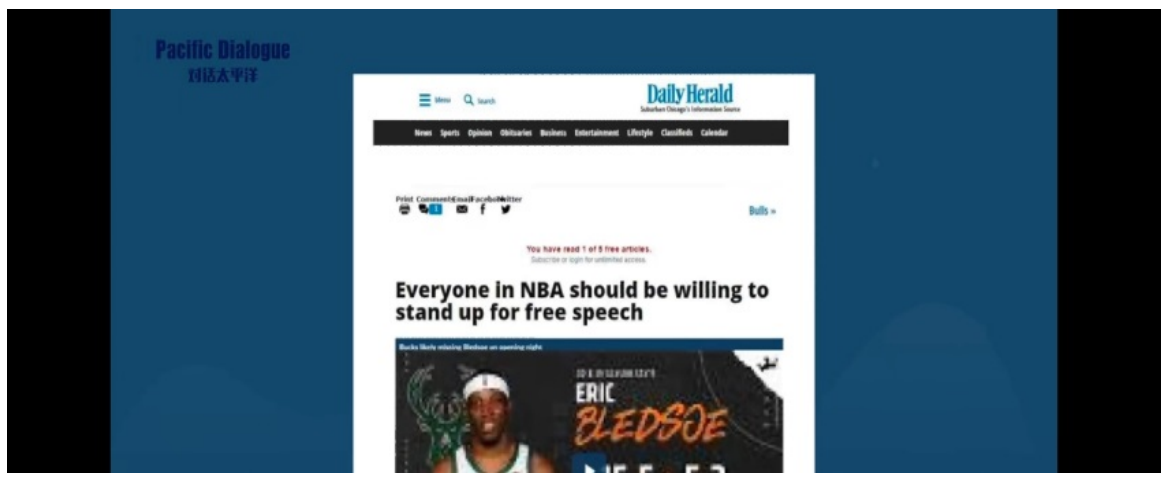

图 11. 每日先驱报。

罚 250 万美元, 并被终身禁赛。当涉及美国社会底线时, 美国怎么就不捍卫 公民的言论自由了呢? 所以故事线 3 得出结论: 西方媒体归因于言论自由是 无稽之谈。

\section{4. 故事线四：从文化互文看冲突}

在文化系统中, 互文性理论将文本和文化(作为一种隐性的存在)很好地 结合在了一起。罗兰 -巴特认为: “任何本文都是互本文; 在一个本文之中, 不同程度地并以各种多少能辨认的形式存在着其他本文: 例如, 先前文化的 本文和周围文化的本文。”从文本角度转移到文化角度, 证实了互文性所关 注的文化传统的影响是两个层面的：即 “先前文化” 和 “周围文化”。前者 
更关注历史的维度, 后者则观察现实。

从纵向的时间角度来看，文化影响主要表现为当代文化与前代文化之间 的对立与统一关系。吸收新历史主义 “历史诗学” 观点的合理性, 将文本置 于一个非文本的历史框架之内，形成相互指涉的互文性描述。历史和文本进 入到了一种互文性的运动之中, 重建文本与历史的交流。图 12 与中国那段屈 辱的历史互动, 唤起了中国观众情感, 提醒了国外观众中国人的社会底线, 即领土完整。19 世纪 40 年代帝国主义向中国发动大规模侵华战争, 强迫清 政府签订一系列不平等条约，侵夺中国香港、台湾，“租借”澳门，蚕食中 国大片国土，迫使中国社会性质发生改变。同时列强在中国强占租借地，干 涉中国内政外交，国弱则民卑。早期的民族意识就此形成。正如习近平总书 记强调大历史观，近代史教会每个中国人保有家国情怀及民族尊严，即国家 兴亡, 匹夫有责的意识。图 12 在这种文化框架中清晰表明立场：中国领土神 圣不可侵犯，犯我中华者，虽远必诛。

图 13 作为整个多模态文本的主背景，中美两国中间连系着一个篮球，与 体育外交形成文化互文，首先从历史背景看，体育外交政策的制定主要依据 当时社会现实情况与整体外交战略的变化，中国体育外交工作的重心始终与 自身外交处境和国际社会环境息息相关。关于中美间的体育外交最显著的就 是 20 世纪 70 年代的乒乓外交。“兵乓外交”之后, 1972 年中美双方发布《上 海公报》，1978 年中美双方发布《中美建交公报》，正式建交。“乒乓外交”

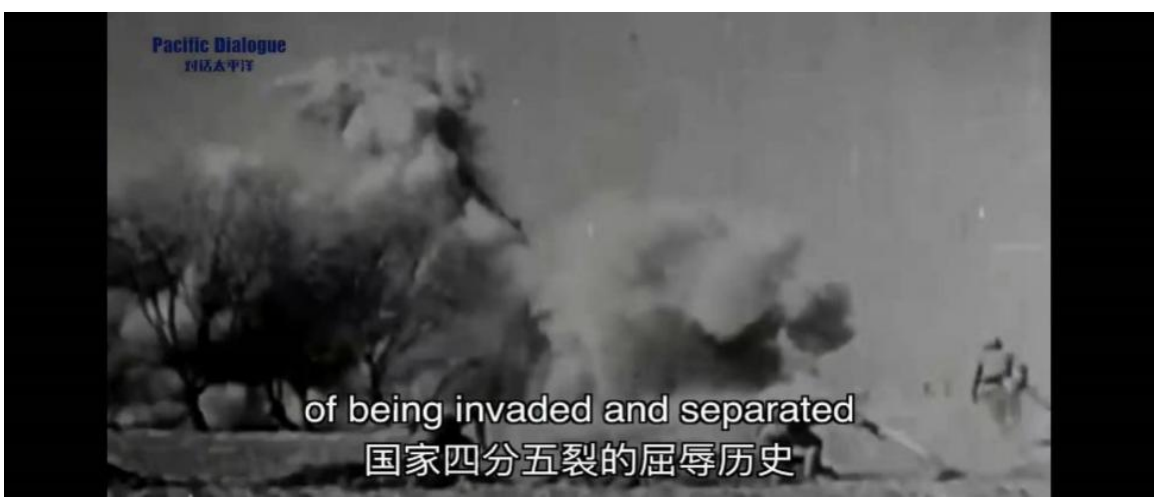

图 12. 历史。

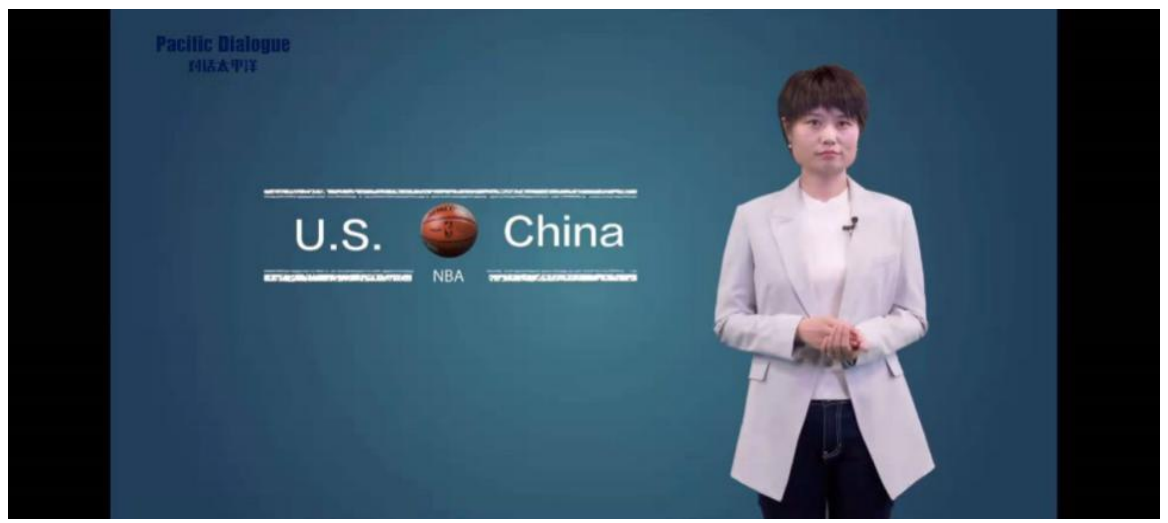

图 13. 体育。 


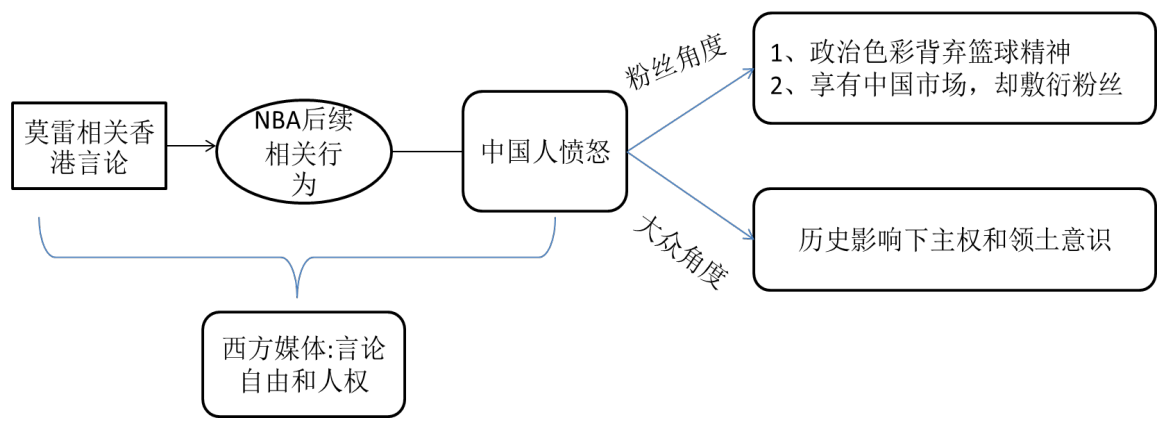

图 14. 故事线。

打开中美关系正常化的大门。近年, 篮球也成为一种文化交流的载体, 促进 中美两国人民互相了解。NBA 风靡中国, 中国的篮球运动员如姚明、易建联, 走出国门加入他们。中国人民也通过这个途径了解美国的文化、了解美国的 体育。但讽刺的是, 这次 NBA 对中国领土的不当言论, 让中国人不得不重新 考量美国人所谓的价值观。美国为了维护自己的霸权主义, 在特朗普领导下 对中国发起贸易战的背景下，体育外交作为中美关系的镜子，折射出现状。

\section{4. 故事线建构与发现}

从上述四条故事线中，我们可以抽出下图这条故事线(图 14): 即 NBA 相 关言论让粉丝不满, 表面原因在于 NBA 态度不诚恳, 深层原因是中国人的家 国和主权意识, 西方媒体将粉丝愤怒解读为言论自由立场不足。

从上述故事线中我们可以抽出几组对立: 宏观来讲, 对于莫雷事件, 中 国认为领土完整是一个国家最基本的原则, 而西方解读为言论自由; 微观看 来: 中国粉丝在爱好和爱国底线之间的抉择; NBA 声明中宣扬体育精神, 却 涉嫌为政治背书; 小球开启中美外交正常化, 现在篮球大球威胁中美人民之 间的社会友好度。在这些对立中, 得出如下发现:

(一)、中国人领土意识, 即家国观念根深蒂固

(二)、中美文化中对言论自由这一概念存在差异

(三)、体育外交是中美关系晴雨表的体现。

\section{5. 发现与讨论}

为了论证这三条发现, 此处采用问卷调查法。问卷的第一部分涉及受试 者对莫雷言论的愤怒以及爱国情感的测试, 第二部分涉及受试者对言论自由 的看法, 第三部分考察对体育外交概念的认可程度。问卷采用了李克特五级 量表, 量表由一组陈述组成, 每一陈述有 “非常同意”、“同意”、“不一 定”、“不同意”、“非常不同意” 五种回答, 分别记为 5、4、3、2、1。调 查对象主要为中国大学生及研究生群体, 具有代表性。调查问卷共回收 160 份, 有效问卷 148 份, 问卷为了结论的有效性, 首先通过 SPSS 对问卷进行信 度检验, 问卷 CroNBAch's Alpha 项数为 0.792 , 量表的信度系数即 CroNBAch's Alpha 一般认为不应低于 0.7 , 本次测量量表的信度系数高于 0.7 , 表明本量表 具有内在一致性, 测量的结果可靠。 
表 1. 调查问卷统计结果

\begin{tabular}{cc}
\hline 爱国情感缘由 & 言论自由程度比较 \\
\hline 莫雷言论刺激下 $81.25 \%$ & 美国较高 $59.38 \%$ \\
传统爱国文化 $92 \%$ & 中国较高 $38.75 \%$ \\
大国观念 $100 \%$ & 二者不可比较 $52.5 \%$ \\
\hline
\end{tabular}

问卷数据(表 1)表明 $81.25 \%$ 的受试者对莫雷言论产生情感波动，约 $92 \%$ 的人认同爱国情感是千百年来固定下来的传统，100\%的被测同意 “先有国再 有家”，作为中国人 $0 \%$ 的测试者不感到自豪，虽然认可程度存在些许差异， 但是数据表明, 代表着中华民族希望的大学生群体一脉相传了中国传统的爱 国主义精神。在对中美言论自由程度的探讨上，59.38\%的受试者认为美国言 论自由程度较高，而仅有 $38.75 \%$ 的受试者认为中国享有同样的程度， $52.5 \%$ 认为与文化紧密相关。通过 SPSS 点二系列相关分析是否英语专业会影响分析 结果, 发现 $\mathrm{p}$ 显著值-0.03 小于 0.05 (在统计学中 $\mathrm{p}$ 是显著性概率, 当概率小 于 0.05 时, 说明假设成立) 说明英语专业的受试者更认为言论自由差异与中美 文化不同相关。对于第三条发现，问卷数据同时显示出受试者认同体育外交 在当代中美关系中发挥着举足轻重的作用, 认为合作包容更有利于彼此间发 展。

所以基于问卷回收结果, 我们得出: 多模态文本 How Much is An NBA Ticket Worth in China Now? 构建的故事线在一定程度上符合当代中国人的 价值观, 问卷调查结果也证实如下结论: “国家”之于中国人神圣不可侵犯, 随着综合国力的不断加强, 中国人的民族自豪感也不断加强; 中美交往需要 注意衡量文化差异; 合作包容的多途径外交手段在中美交往中也发挥着举足 轻重的作用。

\section{6. 结语}

本文从多模态互文出发, 分析出视频中的故事线, 通过问卷调查手段剖 析出结论。希望能够从互文角度为解读多模态文本提供新思路, 也为中美间 交往带来一定的启示。本文的不足在于没有进一步分析受试者文化背景对问 卷结果的影响, 所有的被测都为中国人且受教育程度相对较高。

\section{Conflicts of Interest}

The author declares no conflicts of interest regarding the publication of this paper.

\section{References}

[1] 朱永生. 多模态话语分析的理论基础与研究方法[J]. 外语学刊, 2007(5): 82-86.

[2] van Leeuwen, K.G. (2001) Multimodal Discourse. The Modes and Media of Contemporary Communication. Arnold.

[3] 许晓翠. 多模态话语分析研究综述 [J]. 文化创新比较研究, 2019, 3(29): 84-85.

[4] 李战子. 多模式话语的社会符号学分析 [J]. 外语研究, 2003(5): 1-8+80. 
[5] 张德禄. 多模态话语分析理论与外语教学 $[\mathrm{M}]$. 北京: 高等教育出版社, 2015.

[6] Kristeva, J. (1982) Desire in Language: A Semiotic Approach to Literature and Art. Basil Blackwell, Oxford, 193-194. https://doi.org/10.2307/1772011

[7] 邵长超. 互文性理论的发展阶段、现状与问题分析[J]. 理论界, 2015(11): 124-130.

[8] Palimpsests, G.G. (1977) Literature in the Second Degree. In: Newman, C. and Doubinsky, C., Trans., University of Ne Braska Press, Lincoln NE.

[9] 张春燕. 新媒体语篇的多模态互文性分析一一以一则网络配音视频为例 [J]. 外国 语文研究, 2015, 1(4): 23-31.

[10] 侯建波. 电视公益广告的多模态互文研究[J]. 广西民族大学学报(哲学社会科学 版), 2016, 38(4): 175-178.

[11] van Leeuwen, K.G. (2006) Reading Images. The Grammar of Visual Design. Routledge, $2^{\text {nd }}$ Edition, 177.

\section{Appendix (Abstract and Keywords in Chinese)}

\section{从多模态互文角度分析 “How Much is An NBA Ticket Worth in China Now” 故事线的构建}

摘要: 本文从互文角度对多模态文本“How Much is An NBA Ticket Worth in China Now” 进行剖析, 引用互文理论从外互文、文化互文等角度抽取了 多模态文本中四条故事线, 分析文本中莫雷有关香港言论本身的缺陷及为何 粉丝失望、中国大众不满进而导致 NBA 在中国市场的发展受阻, 进而探寻到 促使故事线发展的根本原因。最终根据故事线得出的发现设计问卷,利用 SPSS 验证信度后，进行问卷调查，验证原因与现实的契合度，从而进一步升华到 结论。即中国人的家国情怀、体育外交的现实作用及言论自由的差异观。

关键词：多模态文本，互文理论，言论自由，家国情怀 